\title{
Systolic anterior motion in hypertrophic cardiomyopathy: a fluid-structure interaction computational model
}

Received: 17 July 2019 / Accepted: 12 February 2021 / Published online: 3 March 2021

(C) The Author(s), under exclusive licence to Springer-Verlag GmbH, DE part of Springer Nature 2021

\begin{abstract}
We present direct numerical simulations for the pathophysiology of hypertrophic cardiomyopathy of the left ventricle of the human heart. This cardiovascular disorder manifests itself through systolic anterior motion (SAM), a drift of the mitral leaflets towards the aortic subvalvular region, sometimes causing ventricular obstruction during systole. This pathology is induced by a combination of factors, including a thickening of the interventricular septum and an elongation of the mitral valve leaflets: we perform a full parametric study to assess their effect on the disease. From our results we observe that SAM occurs when elongated leaflets, hypertrophic ventricles and strong ejection fraction are present at the same time. In contrast, a physiological ventricle with elongated leaflets, an hypertrophic ventricle with physiological leaflets or diseased ventricle and leaflets with a weak ejection fraction do not produce SAM. After verifying that the numerical results are consistent and in agreement with the clinical data from the literature, we virtually test the two standard surgical procedures, leaflet plication and septal myectomy, adopted for the surgical treatment of SAM. For all the considered cases we obtain quantitative confirmation for the reliability of the intraventricular subvalvular pressure drop (or subvalvular pressure gradient for the medical community) as diagnostic indicator of the systolic anterior motion: when this quantity attains the value of $30 \mathrm{mmHg}, \mathrm{SAM}$ of the mitral leaflets is observed, while when this threshold is exceeded the SAM becomes obstructive. On the other hand, in all cases for which SAM is not observed the above pressure drop is always below the threshold.
\end{abstract}

Keywords Fluid-structure interaction $\cdot$ Hypertrophic cardiomyopathy $\cdot$ Systolic anterior motion

\section{Introduction}

Hypertrophic cardiomyopathy (HCM) is a complex but relatively common genetic heart disease that occurs in 1 out of 500 people, [8]. It represents one of the most common causes of sudden cardiac death in people under

Communicated by Jeff D. Eldredge.

V. Meschini $(\bowtie) \cdot R$. Verzicco

Universitá di Roma Tor Vergata, Rome, Italy

E-mail: meschini.valentina@gmail.com

R. Mittal

School of Engineering and Medicine, Johns Hopkins University, Baltimore, USA

R. Verzicco

University of Twente, Enschede, The Netherlands

R. Verzicco

Gran Sasso Science Institute, L'Aquila, Italy 


\section{Normal}

(a)
Hypertrophic cardiomyopathy

(b)

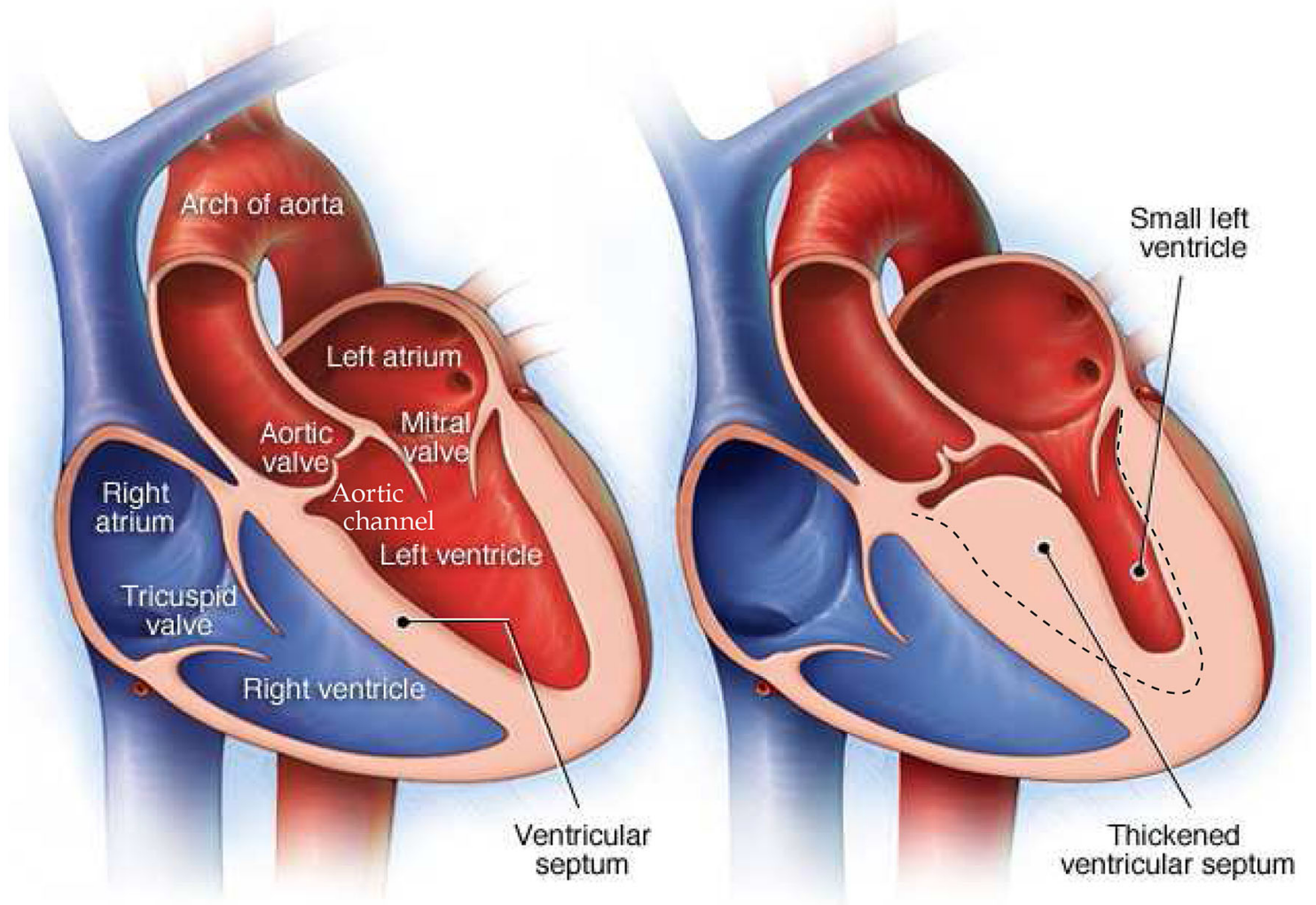

Fig. 1 Schematic of a physiological left ventricle, $\mathbf{b}$ hypertrophic left ventricle. The dashed line in $\mathbf{b}$ is the physiological ventricle shape reported for reference. (Adapted from http://www.mayoclinic.org/diseases-conditions/hypertrophic-cardiomyopathy/ home/ovc-20122102)

30 years, especially in young sportive men [7]. From medical imaging it is found that the HCM occurrence comes with the thickening of the septum between left and right ventricles, with the abnormal growth protruding into the left ventricle (Fig. 1). The hypertrophy of the myocardium can have different severity levels and, in the extreme cases, it can obstruct the blood flow to the aorta and cause sudden death. This is referred to as hypertrophic obstructive cardiomyopathy or HOCM.

It is observed that the majority of patients affected by HCM present elongated mitral leaflets [9] which are more prone to anterior-posterior displacement (flapping) caused by the blood motion. The coupled fluid/tissue interaction is essential to understand the mechanics of the aortic channel obstruction produced by the lateral displacement of the mitral leaflets during systole and indicated as systolic anterior motion (SAM). The pathophysiology of SAM consists of the displacement of the anterior or both mitral valve leaflets towards the intra ventricular septum; the understanding of the causes has been discussed among heart surgeons and cardiologists [15] and still is debated whether it is a push/pull mechanism of the leaflets or a Venturi effect in the outflow tract.

The severity of the obstruction is quantified using the intraventricular subvalvular pressure drop as reference quantity, defined as the pressure difference across the aortic channel: $\Delta p$ values $\simeq 30 \mathrm{mmHg}$ evidence the HCM pathology, while when this threshold is exceeded the anterior displacement of the leaflets can completely obstruct the outflow tract thus producing HOCM [13]. Although HCM is a chronic disease without a known cure, a number of treatments are now available to alter its course. A first possibility is to use beta-blockers to reduce the heart rate and the intraventricular pressure [6]. A more drastic action is to induce a controlled ischemic event of part of the septal myocardium to reduce its volume by necrosis. Finally, the last possibilities are the septal myectomy, that is surgical removal of the abnormal grown portion inside the ventricle, or the mitral leaflet plication (resulting in shortened leaflets) [5]; these two approaches can also be combined together. 
Both of them have drawbacks and advantages and a choice is made for each specific case on the base of medical imaging evidence [12].

From the above considerations it is clear that a computational analysis capable of describing the full HCM dynamics and anticipating the outcome of a particular action would significantly advance the diagnosis process and treatments efficacy of such disease, thus leading to improved outcomes for patients and reduced health care costs.

Motivated by the above arguments in this paper we present the results of a simulation campaign for the fluid/structure interaction (FSI) of the left ventricle and mitral valve aiming at describing the pathophysiology of $\mathrm{HCM} / \mathrm{HOCM}$. A parametric study is performed for different levels of septum hypertrophy starting from the physiological case, as a reference case, up to a severe hypertrophic ventricle, and for two valve leaflets lengths, pathological and physiological. In addition a case with a maximum hypertrophy but with a reduced ventricle pumping efficiency is considered in order to evaluate the effects of the ventricle ejection fraction (later defined) on HCM occurrence. Finally, the surgical procedures of leaflets plication and septal myectomy are simulated and comparisons between the resulting dynamics are performed to assess the efficiency and reliability of these surgical procedures.

The numerical method is not new and it has been already described and verified in [10,17]; in this study, however, we present the results of a novel application of this method to a clinically relevant flow.

The paper is organized as follows: in the next section the numerical set-up, the numerical algorithm with the flow and structure parameters are given. In Sect. 3 the results obtained for different system parameters are presented and analysed. The closing remarks are given in the final section.

\section{Computational model}

\subsection{The set-up}

The present computational model, in principle, could cope with any ventricle geometry and, therefore, also with patient specific cases. Nevertheless, this study is aimed at understanding the general flow and tissue dynamics involved in HCM/HOCM and not at performing patient specific analyses; accordingly, all the geometrical and physical details of the analysed systems have been obtained from averages of high-resolution clinical images and medical atlas [18] so that the obtained configurations can be considered as representative of an adult human and not of a particular individual $[1,14,19]$. The final physiological configuration results in an 'idealized' structure of constant thickness $8 \mathrm{~mm}$ and the two characteristic lengths of $44 \mathrm{~mm}$ (diameter) and $55 \mathrm{~mm}$ (long axis). All these values lay in the range of available clinical measurements/data for a physiological ventricle $[3,19,21]$.

In addition, our reference geometries have been used also to produce silicone rubber replicas of the ventricles that have been employed for validation laboratory experiments (see [10]).

In Fig. 2 three different ventricle geometries are shown and in all of them we have taken as a reference length the mitral diameter $D=2.4 \mathrm{~cm}$ giving a geometric area of $A \simeq 4.5 \mathrm{~cm}^{2}$. The physiological left ventricle (blue shaded) has an end-systolic volume $V_{0} \simeq 58 \mathrm{~cm}^{3}=4.3 D^{3}$ with no thickening of the septum. A second ventricle with a first level of hypertrophy (green shaded) has a smaller volume $V_{1} \simeq 39 \mathrm{~cm}^{3}=2.9 D^{3}$ obtained by displacing the left side of the septum into the left ventricle by a distance $t_{1}=0.75 D$. A third ventricle with a severe thickening $\left(t_{2}=D\right)$ has an even smaller volume $V_{2} \simeq 27.4 \mathrm{~cm}^{3}=2.2 D^{3}$ (red solid). The ventricles have a local thickness $\theta$ (later specified) that is uniform for the healthy geometry while it is position dependent for the pathological configurations.

The contraction and expansion of the ventricle is not driven by the propagation of an electrical stimulus as in the human heart but is rather controlled by an inflow/outflow function, as shown in Fig. 3. This function is composed of a first inflow phase, the diastole, where $U(t)$ is positive and the fluid is pumped into the ventricle through the mitral duct. In the second outflow phase, $t \geq 0.65, U(t)$ is negative and the fluid can leave the left ventricle both through the mitral and aortic ducts. However, owing to the counter pressure, the mitral valve quickly closes and the leaflets start prolapsing into the channel because of the suction. This phenomenon (closure and prolapse) occurs in short time $(\approx 0.05)$ and, when the leaflet have reached the maximum allowed vertical displacement (set by the kinematic constraint), the mitral suction is stopped and the flow can leave the ventricle only through the aortic channel.

The contraction and expansion of the ventricle is not driven by the propagation of an electrical stimulus, as in the human heart, but it is rather controlled by an imposed inflow/outflow function, as in Fig. 3. This 
(a)

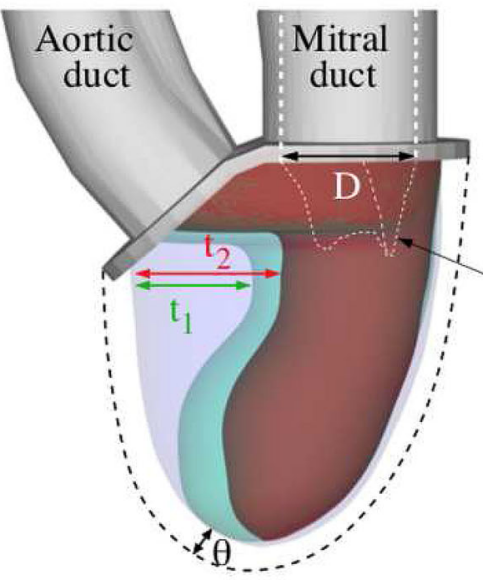

(b)

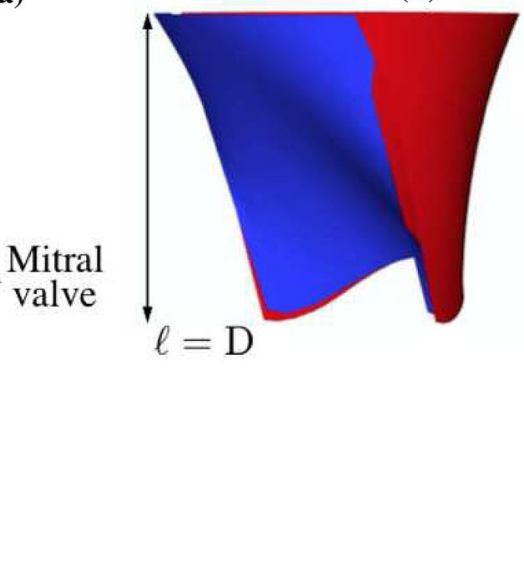

(c)

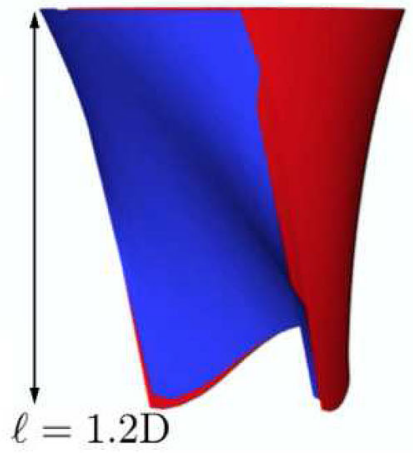

Fig. 2 a Three geometries for the left ventricle: physiological ventricle (blue shaded), a first level of hypertrophy left ventricle, $t_{1}$ (green shaded), a second level of hypertrophy left ventricle, $t_{2}$ (red solid). The black dashed line is reported to evidence the local thickness of the myocardium, $\Theta$. The white dashed shape of the mitral valve is reported with the reference length of the mitral diameter D. b, c Anterior (blue) and posterior (red) leaflets of the mitral valve in the b physiological and $\mathbf{c}$ pathological HCM case

profile is composed of a first inflow phase, the diastole, where $U(t)$ is positive and the fluid is pumped into the ventricle only through the mitral duct. In the outflow phase, $t \geq 0.65, U(t)$ is negative and the fluid leaves the left ventricle both through the mitral and aortic ducts with the same velocity. However, the mitral valve quickly closes, owing to the counter pressure, and its leaflets seal thus preventing further mitral leakage. This phenomenon occurs in a short time $(\approx 0.05)$ and the total mitral regurgitation is of the order of $5 \%$ of the cardiac output which is considered within the physiological range. Even if the mitral valve is closed, being the leaflets deformable, they can still move towards the mitral duct if the outflow is not halted; for this reason, when the leaflets have reached the maximum allowed vertical displacement (set by a kinematic constraint), the mitral suction is stopped and the flow can leave the ventricle only through the aortic channel.

In all the simulated cases, except for one, our mitral valve does not include chordae tendineae which connect the tips of the mitral leaflets to the myocardium through the papillary muscles and prevent the leaflets from everting into the left atrium during systole. Since they exert mainly a vertical pulling action, they are mimicked by a kinematic constraint that limits the leaflets vertical displacement (see [10] for more details). This is justified since in a separate study [11] it has been shown that the inclusion of chordae tendineae in the model does not appreciably affect the global dynamics of the intraventricular flow and neither prevent nor determine SAM. It is important to note that our kinematic constraint does not limit in any way the lateral motion of the leaflets that is entirely determined by the fluid/structure interaction as part of the solution.

Nevertheless, in order to better ascertain the role of chordae tendineae, we have simulated also one case in which we endow the mitral valve with them and the left ventricle with papillary muscles, to which the chordae are attached, for the case of medium hypertrophy of the left ventricle. The aim is to understand whether the presence of chordae tendineae affects the overall dynamics of HOCM and especially the occurrence of SAM or, as we expect, the main features of HOCM and the intraventricular flow dynamics are only marginally influenced by their presence.

It is worth mentioning that we do not model explicitly the aortic valve, since it regulates the outflow of the ventricle and has a minor impact either on the intra-ventricular flow and on the mitral valve dynamics. Accordingly, in order to reduce the computational load, we mimic its opening and closing by using a porous medium in the aortic duct, with a porosity tending to zero during diastole and to infinity during systole, as already explained in [10].

The pumping efficiency of the left ventricle has been quantified by its ejection fraction $(E F)$; let $V(t)$ be the ventricle volume during the cardiac cycle with a maximum $V_{M}$ at the end of the diastolic phase, when the myocardium is fully expanded, and a minimum $V_{\mathrm{m}}$ at the end of systole, when the myocardium ends its contraction; the difference $V_{\mathrm{M}}-V_{\mathrm{m}}$ is the blood volume ejected from the ventricle in one cycle and the ejection fraction is defined as $E F=\left(V_{\mathrm{M}}-V_{\mathrm{m}}\right) / V_{\mathrm{M}}$. This parameter has some variation among individuals but values $55 \% \leq E F \leq 70 \%$ are considered physiological; in this study we always use the value $E F=60 \%$ except 


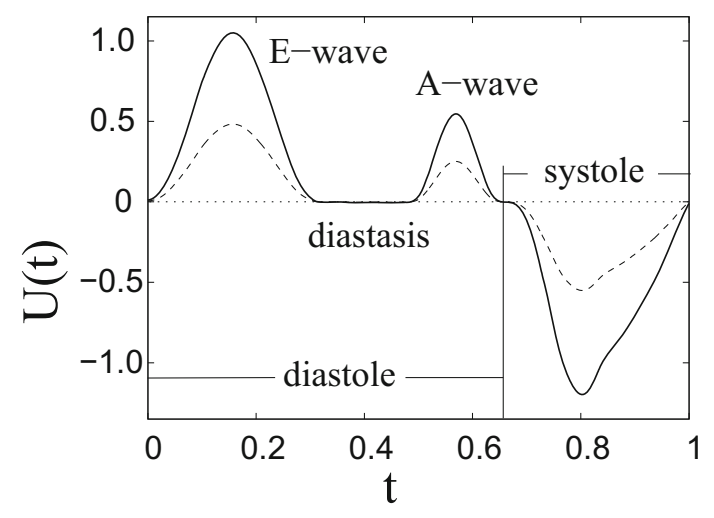

Fig. 3 Time evolution of the flow rate function $U(t)$ over a period. Solid lines curve for ventricle ejection fraction $E F=60 \%$, dashed lines $E F=40 \%$

for one case in which we impose $E F=40 \%$ in order to assess the effect on the ventricle ejection fraction on the SAM.

The mitral and aortic ducts together with the valve planes (in grey in Fig. 2) are modelled as a rigid structure that is used as a frame to anchor the deformable ventricle and the mitral valve. We note that this arrangement is different from that of the human heart in which the ventricle apex is fixed and the valvular plane moves. This difference, however, has already been discussed and quantified in [10] where the main conclusion was that the pressure gradient introduced by the ventricle valvular plane translation is at lest one order of magnitude smaller than that produced by the inflow/outflow dynamics.

The leaflets of the mitral valve are generally found to be elongated in case of HCM [9]; in Fig. 2b, c, the physiological (Fig. 2b) and the pathological (Fig. 2c) geometries are reported for comparison. Since we use deformable membranes, a crucial point is the modelling of the contact mechanism between the leaflets during their motion. We model each structural element by a triangular mesh whose nodes are evolved in time according to the equations of membranes, as already detailed in $[10,17]$. To model the contact of deformable structures we use an array that is null in the fluid and assumes a specific integer value, different for each body, in the cell occupied by the nodes of a specific body. The value in the cell is updated every time step and if a triangle ends up in a non-null cell, meaning that the position is already occupied by another triangle, then the velocities of both surface elements are set to their average so that they can still freely move together in space but cannot compenetrate. The same procedure is used to model the contact between the leaflet and the ventricle, but once a triangle ends up in a non-null cell the velocities are reversed in sign to induce a sort of rebound. We have found that this approach is computationally efficient and it can handle the valve closing without prescribing a coaptation line or surface. It prevents the compenetration of the membranes but, at the same time, it allows the lateral motion of the valve leaflets even when they are coapted together.

\subsection{Numerical method}

The numerical scheme is that described in $[2,10,17]$ and it consists of a Navier-Stokes solver two-way coupled with a structure solver for membranes. We refer to the above references for a detailed description of the method, the convergence checks and the experimental validation of the model; here we report only the main features.

The motion of the blood is modelled as a Newtonian, incompressible and viscous fluid, described by the Navier-Stokes equations which, in non-dimensional form, read

$$
\begin{aligned}
& \frac{\partial \mathbf{u}}{\partial t}+\mathbf{u} \cdot \nabla \mathbf{u}=-\nabla p+\frac{1}{\operatorname{Re}} \nabla^{2} \mathbf{u}+\mathbf{f}, \\
& \nabla \cdot \mathbf{u}=0,
\end{aligned}
$$

where $\mathbf{u}$ is the velocity, $p$ is the pressure and $\mathbf{f}$ is a body force term related to the immersed boundary technique. It has to be mentioned that even if the blood nature is non-Newtonian owing to its relevant corpuscular volume fraction (the haematocrit), here it behaves as a Newtonian fluid since it flows in large vessels and cavities. In a recent paper [10] the Carreau Yasuda shear-thinning model [16] has been used to assess the non-Newtonian 
effects showing that indeed there is substantial agreement with the Newtonian fluid model either for the ventricular flow and for the tissue dynamics. The latter is solved using an interaction potential approach introduced by [20] and applied to the present phenomena by [2,10,17]. The surfaces of the ventricle and mitral valve are discretized using triangular elements. The system appears as a complex network which deforms according to the external forces and internal reactions, given by elastic, bending, area and volume energy which is stored into the system. The properties of the structure, modelled as membranes, are computed using the Van Gelder model, [22].

Two different immersed boundary methods are employed to compute the influence of the structure on the fluid and vice versa. For the moving and deformable bodies we use the moving least square approach of [23] since, despite the high computational cost, it gives smooth local loads. In contrast, for rigid structures, the inexpensive direct-forcing method of [4] is adopted.

A loose-coupling fluid/structure interaction (FSI) algorithm is used to compute the synergistic interplay of fluid and tissue dynamics. In order to keep the integration stable the procedure relies on time substepping to advance the structure deformation: namely, starting from the flow and structure at the time $t^{n}$, the flow at the new time $\left(t^{n+1}=t^{n}+\Delta t\right)$ is computed; then, the structure is advanced with a time substep $\Delta t_{s}=\Delta t / S$, $\mathrm{S}$ being the number of substeps, until the time $t^{n+1}$ is reached, using the hydrodynamic loads interpolated between old and new times, $t^{n}$ and $t^{n+1}$, a typical value of $S=100$ is used in all the simulations. This algorithm calls the flow solution and the IB procedure only once per time step and requires multiple calls only of the structure solver, which, being much faster than the rest, does not penalize the overall performance.

\subsection{Simulation parameters and convergence checks}

As discussed in [10], after a careful grid independence study a grid resolution of $129^{3}$ is used in all simulations performed in this paper. In order to maintain the optimal ratio between the Lagrangian (triangular) and the local Eulerian meshes of about $70 \%$, [2], the left ventricle has been discretized by $3 \times 10^{4}$ triangles while the two leaflets of the natural mitral valve have a total of $8 \times 10^{3}$ triangles. Both, tissue properties and flow parameters are made dimensionless using the mitral orifice diameter $D=24 \mathrm{~mm}$, the inflow velocity $U \simeq 0.75 \mathrm{~m} / \mathrm{s}$ at the peak of the E-wave and the fluid density $\rho=1000 \mathrm{~kg} / \mathrm{m}^{3}$. Considering a blood with kinematic viscosity $v=4.5 \times 10^{-6} \mathrm{~m}^{2} / \mathrm{s}$, the resulting Reynolds number is $\mathrm{Re}=4200$. A heart rate of $72 \mathrm{bpm}$ has a period $T=0.833 \mathrm{~s}$ that corresponds to $T_{a d}=25$ non-dimensional time units.

This Reynolds number might appear too large for a grid resolution of $129^{3}$ and, for the present set-up, this would be indeed the case if $\operatorname{Re}=4200$ were sustained throughout the whole heart cycle. However, $R e$ is computed using the peak velocity of the E-wave which is attained only in one instant and the cycleaverage r.m.s. Reynolds number is below 700. Another relevant observation is that the flow is pulsatile and the time during which the Reynolds number remains large is too short to allow the energy to cascade down to the Kolmogorov scales. This implies that the standard estimates (used for statistically steady, homogeneous isotropic turbulence) do not apply directly to the present flows and only the grid refinements, described in [10] can assess the adequacy of the mesh.

Following [10], for the ventricle an incompressible homogeneous elastic material with elasticity modulus $E_{v}=20 \mathrm{kPa}$ and Poisson ratio $v_{\mathrm{p}}=0.5$ with a local thickness $\theta$ ranging from $6 \mathrm{~mm}$ up to $25 \mathrm{~mm}$ (in the severely hypertrophic septum) has been used. We wish to stress that the chordae configuration of this additional case is highly idealized and it completely misses the active contraction of the papillary muscles. The aim of this simulation was only to show that SAM is not significantly modified by the chordae that are anyway loose during late systole. For the mitral valve leaflets, instead, the elasticity modulus has been chosen $E_{l}=3 \mathrm{MPa}$ while the (uniform) thickness set to $\theta_{l}=2 \mathrm{~mm}$. We are aware that this might be a too simplistic approximation since the tissues of the heart are orthotropic, hyperelastic and they exhibit even visco-elastic properties. Indeed the present method could simulate this kind of materials [2] provided all the material parameters were known; given the considerable uncertainty characterizing the mechanical properties of the biological soft tissues and considering that the their internal stresses are not the main focus of the paper, we have decided to avoid this difficulty in the present first study of HCM. We also wish to stress that in [10] it has been shown that the tissue dynamics is dominated by the pressure forces and the added mass, therefore their kinematics is not very sensitive on the specific values of the material properties (if they are assigned within 'reasonable' values). Needless to say, should the focus of the investigation be the values and the distributions of the tissue internal stresses, not only the material properties but also their constitutive relations would be of utmost importance.

The time integration has been performed at constant Courant number $\mathrm{CFL}=0.2$ with a dynamic time stepping that adjusts during the integration so to keep the CFL constant. 


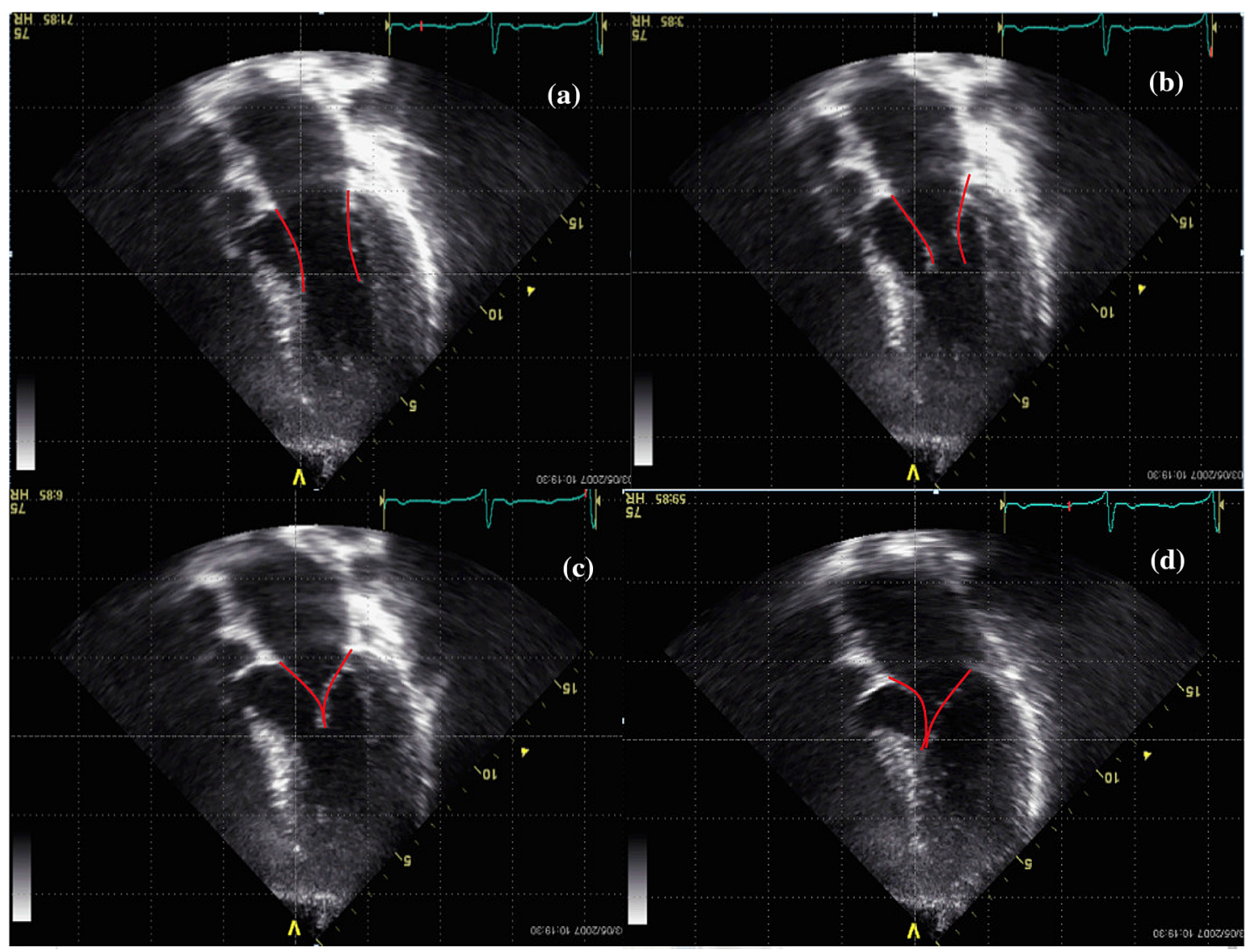

Fig. 4 Snapshots of the ecocardiography of a patient affected by HCM in selected instants of the cardiac cycle: a peak of the E-wave, $\mathbf{b}$ peak of the A-wave, $\mathbf{c}$ mid-systole, $\mathbf{d}$ end-systole

For the validation of the code we refer to $[10,11]$ in which two different one-to-one experiments are performed for the validation of the flow inside the ventricle, the structure deformation and the mitral valve dynamics; comparisons of the numerical results and experimental measurements are performed for several quantities like the time-dependent area of the mitral valve, pointwise and two dimensional velocity measurements as well as ventricle deformation and mitral leaflets dynamics.

\section{Results}

Before discussing the results of the numerical simulations, we wish to describe the main features of the HCM as they appear from medical imaging (ecocardiography). In Fig. 4 four sequential snapshots are extracted, at representative instants of the heartbeat, to illustrate the flow dynamics, the ventricle and mitral valve motion.

The blood from the left atrium flows into the left ventricle through the mitral orifice, the valve opens until the peak of the E-wave is reached and the maximum opening position is attained (Fig. 4a). The A-wave, produced by the left atrium active contraction, generates a new mitral jet that opens again the mitral valve as shown in Fig. 4b. During systole the contraction of the ventricle increases, the local pressure closes the mitral valve (Fig. 4c) and opens the aortic valve thus allowing the blood to flow into the aorta. Because of the pathologically thickened septum, however, the blood undergoes strong accelerations in the aortic subvalvular region that produce abnormal local low pressures owing to Bernoulli effects. As a consequence the mitral leaflets experience an anomalous lateral pressure gradient that 'sucks' them towards the thickened septum (Fig. 4d) thus impeding the correct ventricle emptying; for severe pathologies the mitral leaflets can even come in contact with the thickened septum and impede the ventricular outflow.

We start from these clinical observations as a guideline for a numerical study to investigate under which conditions this pathological behaviour is observed. In order to explore the variety of behaviours we consider 
Table 1 Details of the simulated cases

\begin{tabular}{lllll}
\hline Case & $V_{\text {min }}$ & $\ell$ & $E F(\%)$ & $S P D$ \\
\hline$\# 0$ & $4.3 D^{3} \simeq 58 \mathrm{~cm}^{3}$ & $1.0 D$ & 60 & $2.0 \simeq 10.0 \mathrm{mmHg}$ \\
$\# 1$ & $2.9 D^{3} \simeq 39 \mathrm{~cm}^{3}$ & $1.2 D$ & 60 & $6.1 \simeq 30.5 \mathrm{mmHg}$ \\
$\# 2$ & $2.2 D^{3} \simeq 27 \mathrm{~cm}^{3}$ & $1.2 D$ & 60 & $6.4 \simeq 34.0 \mathrm{mmHg}$ \\
$\# 3$ & $4.3 D^{3} \simeq 58 \mathrm{~cm}^{3}$ & $1.2 D$ & 60 & $4.2 \simeq 20.8 \mathrm{mmHg}$ \\
$\# 4$ & $2.2 D^{3} \simeq 27 \mathrm{~cm}^{3}$ & $4.2 D$ & $5.4 \simeq 27.0 \mathrm{mmHg}$ \\
$\# 5$ & $2.2 D^{3} \simeq 27 \mathrm{~cm}^{3}$ & $1.0 D$ & 60 & $3.8 \simeq 19.0 \mathrm{mmHg}$ \\
$\# 6$ & $2.9 D^{3} \simeq 39 \mathrm{~cm}^{3}$ & $1.2 D$ & 60 & $4.0 \simeq 20.0 \mathrm{mmHg}$
\end{tabular}

$V_{\min }$ is the ventricle volume at the end of systole, $\ell$ is the length of the mitral valve leaflets as indicated in Fig. 2, $E F$ the ejection fraction, $S P D$ the aortic subvalvular pressure drop defined as the maximum pressure difference in the ventricle at peak systole in the aortic subvalvular region

the following configurations: a mild hypertrophic ventricle, with an ejection fraction $E F=60 \%$ and elongated leaflets (Sect. 3.1); a second hypertrophic ventricle with a more severe septum thickening and elongated leaflets (Sect. 3.2). By two additional cases we show that the thickening of the septum or the elongated mitral leaflets alone are not enough to produce systolic anterior motion; accordingly, a healthy ventricle (with no hypertrophy) with elongated mitral leaflets (Sect. 3.3) and a severe hypertrophic ventricle with elongated leaflets but with a reduced ejection fraction of $E F=40 \%$ (Sect. 3.4) are simulated. Finally, the surgery of leaflets plication and septal myectomy are simulated for the case of the most severe hypertrophy and their effects on the reduction of the SAM are analysed (Sect. 3.6).

A table with a summary of the considered cases is given in Table 1 together with the intraventricular subvalvular pressure drop $S P D$, defined as the maximum pressure difference within the ventricle in the aortic channel. The first simulation (Case \#0) is that of a healthy configuration, taken from [10], which will be used as reference to assess the pathologic effects.

\subsection{Case \#1: Medium hypertrophy and elongated leaflets}

Here we discuss the results for a case of a mild hypertrophic ventricle with an end systolic volume of $V_{\min }=$ $2.9 D^{3}$ which is about two-thirds of the physiologic value. The mitral valve presents an elongation of the leaflets $\ell=1.20 D$ as sketched in Fig. 2c. In Fig. 5 we show four instantaneous snapshots of the vertical velocity in the symmetry plane, that is the $x-z$ plane at $y=0$, and velocity vectors at the same representative instants of the cycle as in Fig. 4. The blood enters the ventricle through the mitral duct according to the inflow function of Fig. 3. Initially the asymmetric leaflets deviate the mitral jet towards the posterior wall but, as the flow rate increases, the valve opens wider and the jet sweeps all the ventricle wall up to the apex (Fig. 5a). This produces a strong flow recirculation that is strengthened by the second jet of the A-wave (Fig. 5b). As systole starts, the ventricle contracts and the increasing intraventricular pressure closes the mitral valve thus allowing the flow only through the aortic duct (Fig. 5c). During this phase, however, the thickened septum reduces the section of the aortic channel, in turn, inducing abnormal high velocity and low sub-valvular aortic pressure; this results in the migration of the mitral leaflets towards the aortic channel thus producing SAM (Fig. 5d).

Further evidence of the described dynamics is given in Fig. 6 showing the comparison of the pressure at the peak systole for the present case and for the analogous quantity for a healthy configuration. In both simulations, the flow rate at the outflow of the aortic duct is the same since it is prescribed through the function of Fig. 3. It is immediately evident that a higher ventricular pressure is found in the hypertrophic case owing to the different ventricle geometry and to the throat geometry in between the septum and the mitral leaflets. This has the effect of producing a streamwise pressure gradient, responsible for the flow losses, and a lateral pressure gradient that induces SAM. Another important difference is in the closure of the valve whose leaflets are more coapted in the healthy than in the hypertrophic case.

In order to characterize the SAM we describe in Fig. 7a the horizontal dynamics of the mitral valve during a heart beat by analysing the time evolutions of the anterior $\bar{X}_{\mathrm{a}}$ and posterior $\bar{X}_{\mathrm{p}}$ leaflets centroids (Fig. 5); for comparison also the same quantities for a healthy ventricle and a physiological mitral valve are reported from [10]. In the initial part of the cycle, as the E-wave increases in strength, the valve leaflet spread as highlighted by the decreasing value of $\bar{X}_{\mathrm{a}}$. It can be noted that the posterior leaflet has in comparison a smaller excursion of $\bar{X}_{\mathrm{p}}$ owing to the opposite membrane curvature that stiffens the structure. During diastasis $0.3 \leq t \leq 0.5$, in 
(a)

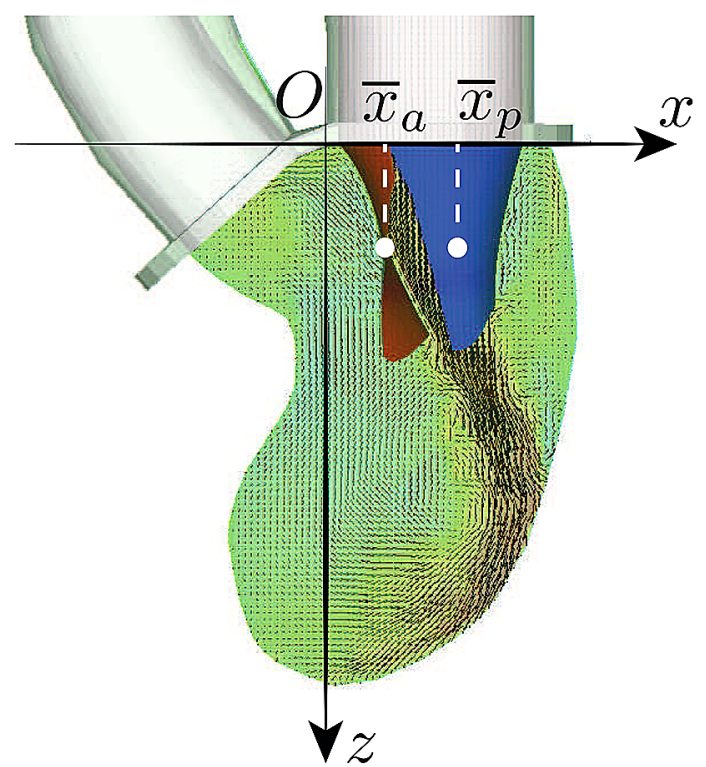

(c)

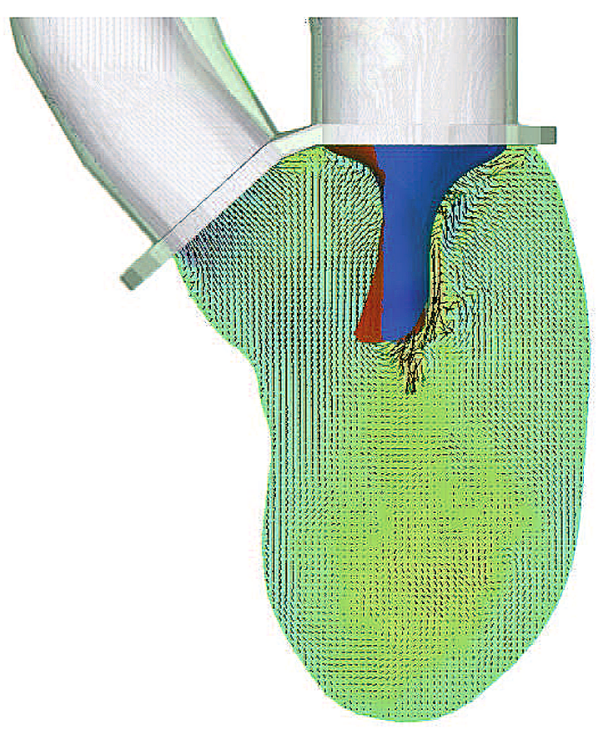

(b)

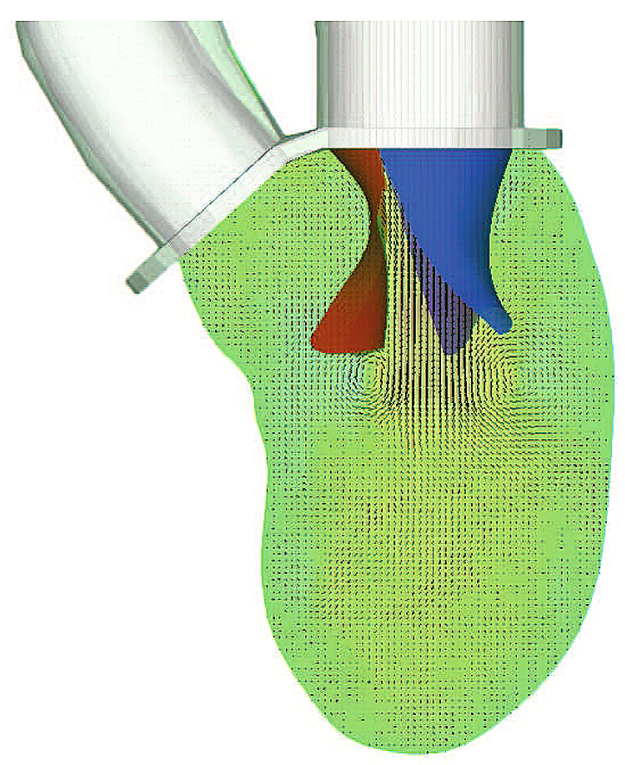

(d)

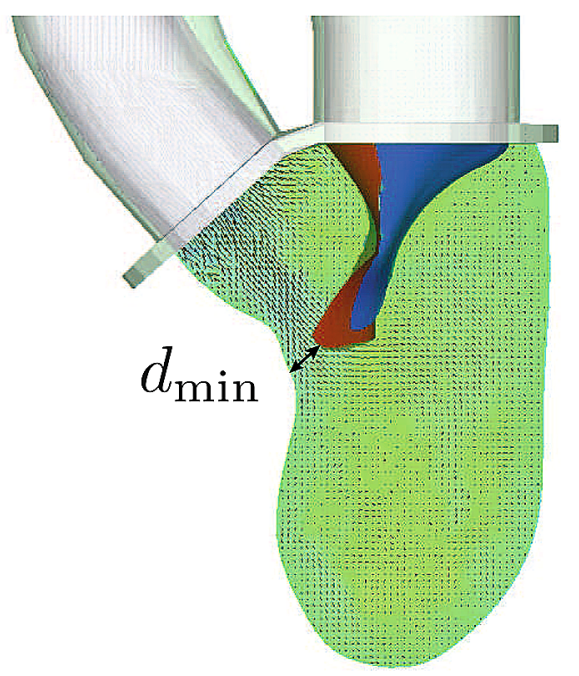

Fig. 5 Snapshots of the velocity vector in the $x-z$ symmetry plane and contours of vertical velocity at $E F=60 \%$ at different instants of the cardiac cycle for Case \#1: a peak of E-wave, $\mathbf{b}$ peak of the A-wave, $\mathbf{c}$ mid-systole, $\mathbf{d}$ end-systole. The white bullets on the mitral leaflets of panel a indicate the positions of the centroids whose $x$-coordinates are reported on the $x$-axis. The distance $d_{\min }$ is the minimum distance between the mitral leaflets and the ventricle septum at the end of diastole

absence of mitral flow, the valve should partially reduce the leaflets spreading and this is indeed the case for the healthy reference case showing increasing $\bar{X}_{\mathrm{a}}$ and decreasing $\bar{X}_{\mathrm{p}}$. For the present pathologic configuration, in contrast, the altered ventricular flow produces a further spreading of mitral leaflets even within the diastasis and this spreading is maintained during the A-wave. For $t<0.65$ the systole starts and the ventricle contracts thus producing an overpressure that closes the mitral valve and brings its leaflets in contact. This is true both for the physiological and the pathological case and Fig. 7a indicates that at $t \simeq 0.75$ the coaptation is complete. In the former case, however, after $t=0.75$ the leaflets remain in their position until, in the successive heartbeat, a new A-wave will open them. In the latter case, in contrast, the low-pressure region induced by the abnormal flow velocity in the aortic channel produces a suction force that causes the anterior motion of the leaflets during 


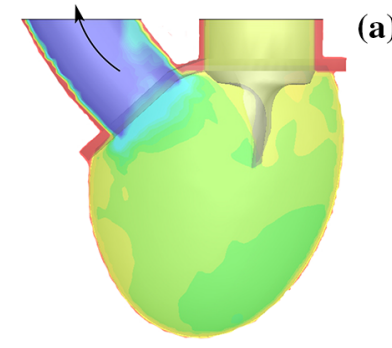

(a)

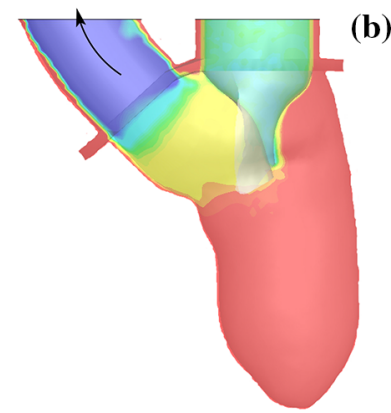

Fig. 6 Snapshots of the pressure field in the $x-z$ symmetry plane at the peak systole: a healthy physiologic case (Case \#0, data from [10]), b Case \#2, hypertrophic ventricle with elongated leaflets. Pressure contours range from $p_{\min }=0$ (blue) to $p_{\mathrm{Max}}=7.5$ (red)

(a)
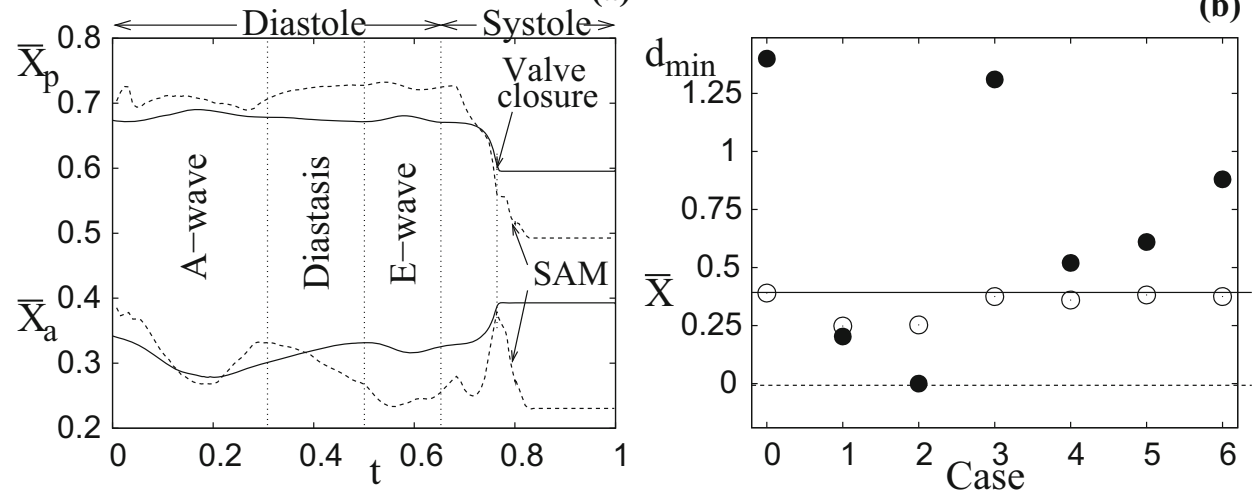

Fig. 7 a Time evolution of the anterior $\bar{X}_{\mathrm{a}}$ and posterior $\bar{X}_{\mathrm{p}}$ leaflet centroid for mild ventricle hypertrophy (Case \#1) (thick dashed lines) and physiological configuration (Case \#0) (solid lines) cases. b Mean mitral leaflets position $\left(\bar{X}=\left(\bar{X}_{\mathrm{a}}+\bar{X}_{\mathrm{p}}\right) / 2\right.$ (open circle) and minimum anterior mitral leaflet-ventricle septum $d_{\min }$ (filed circle) after the closing of the valve. The line dashed lines indicated the contact between anterior mitral leaflet and ventricular septum, and the line solid lines gives the reference value for the physiological $d_{\min }$

systole. In this specific case SAM continues until, at $t \simeq 0.85$, both leaflets attain their leftmost position and remain in that configuration up to the end of the cycle.

The described dynamics is summarized, for the present and all the considered cases, in Fig. $7 \mathrm{~b}$ by the mean mitral valve centroid $\bar{X}=\left(\bar{X}_{\mathrm{a}}+\bar{X}_{\mathrm{p}}\right) / 2$ and the minimum mitral leaflets/ventricular septum distance $d_{\min }$ (see Fig. 5), both at the end of systole. These quantities for the healthy reference configuration (Case \#0) show that $\bar{X} \simeq 0.4$ and $d_{\min } \simeq 1.4$ indicating the absence of SAM and a cleared aortic subvalvular region. For the present case, in contrast, we observe a smaller value of $\bar{X}$ that confirms the anterior drift of the mitral leaflets and a substantially reduced $d_{\min }$ evidencing a partial obstruction of the outflow ventricle tract.

In the clinical practice a quantity used to characterize the HCM pathology is the intraventricular subvalvular pressure drop $(S P D)$ defined at the beginning of Sect. 3 as the pressure difference upstream and downstream the aortic subvalvular region. This quantity is also referred to as 'subvalvular pressure gradient' by the medical community and it is widely used in this context $([8,13])$ owing to its relative simplicity and correlation with the pathology. Cardiac surgeons define obstructive the hypertrophic cardiomyopathy if $S P D^{*}>30 \mathrm{mmHg}$ and non-obstructive if it is $\simeq 30 \mathrm{mmHg}$. In our model this threshold corresponds to the non-dimensional value $S P D \approx 6$ and we have obtained $S P D=6.1 \simeq 30.5 \mathrm{mmHg}$ which is just above the limit for the SAM pathology to deserve treatment, as confirmed by the fields of Fig. 5.

\subsection{Case \#2: Severe hypertrophy and elongated leaflets}

The role of the septum thickening on the pathophysiology of HCM is investigated here by running a simulation with the same set-up as the previous section except for the ventricle geometry which has a more pronounced 


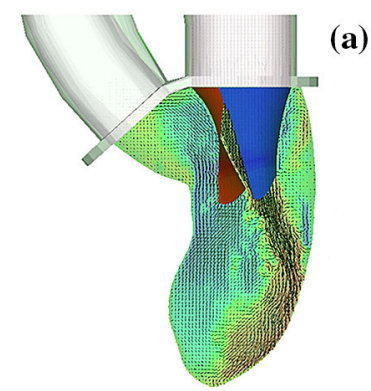

(a)

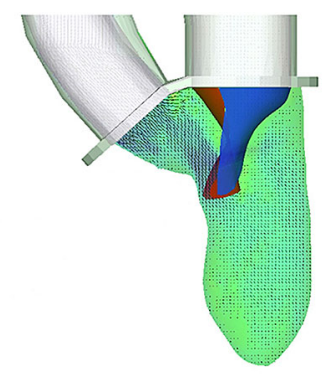

(b)

Fig. 8 The same as Fig. 5, but for a ventricle geometry with a more severe thickening (case \#2): a peak of E-wave, b end-systole
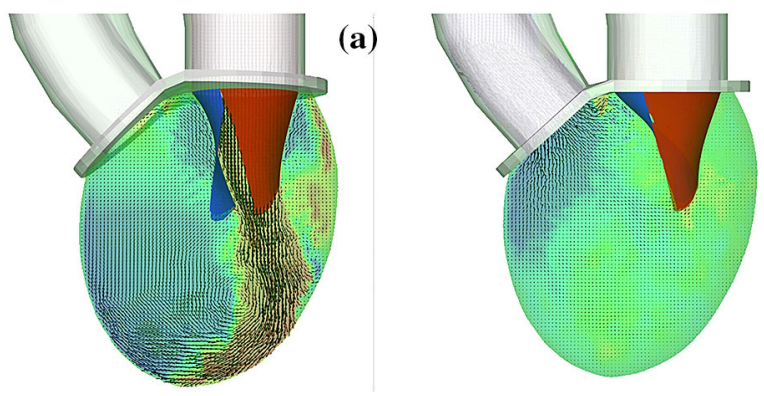

(b)

Fig. 9 The same as Fig. 5 but with the geometry of a physiological left ventricle with elongated leaflets: a peak of E-wave, b end-systole

hypertrophy (red shape of Fig. 2). This severely hypertrophic ventricle is characterized by an initial volume $V_{2}=2.20 D^{3}$, corresponding to about half the physiological value. In Fig. 8 two snapshots, at the peak of the E-wave $(t=0.15)$ and at the peak systole $(t=0.8)$, of the velocity vectors and the field of vertical velocity in the symmetry plane are reported showing a dynamics similar to the previous case. Also in this case, after the mitral valve closure $(t \simeq 0.75)$ the coapted leaflets start the anterior drift even if, owing to the more severe pathology, they come in contact with the ventricular septum $(t \simeq 0.85)$. From Fig. $7 \mathrm{~b}$ we note that $\bar{X}$ has a value very close to that of Case \#1 which might look counterintuitive on account of the more severe septum hypertrophy. However, the fact that $d_{\min } \simeq 0$ indicates that the contact of the leaflet with the septum has occurred and this evidences the obstructive nature of the pathology.

Also for this flow the subvalvular pressure drop has been computed obtaining the value of $S P D \approx 6.4$, corresponding to $\simeq 34 \mathrm{mmHg}$, which is beyond the threshold value and consistently producing obstructive effects.

\subsection{Case \#3: Physiological ventricle with elongated leaflets}

According to [8] the thickening of the ventricular septum is often accompanied by an abnormal length of the mitral valve leaflets and it is not clear whether both factors are necessary to cause SAM or one of them alone is enough to produce the adverse effect. Here we analyse the flow in a physiological ventricle (Fig. 2a) but with a mitral valve having the same elongated leaflets (Fig. 2c) as in cases \#1 and \#2. Apart from the ventricle geometry, all the other flow parameters are the same as for the two previous sections.

The snapshot of Fig. 9a shows that at the peak of the E-wave, despite the pathological valve, a mitral jet is still produced with enough strength to reach the ventricle apex and prevent the fluid stagnation. The most important feature is, however, that, during systole (Fig. 9b), after the mitral valve closure, the aortic channel is not restricted by the septal growth and consequently the mitral leaflets do not show the anterior drift. Confirmation of this behaviour comes from Fig. $7 \mathrm{~b}$ where the mean leaflets position $\bar{X}$ and the distance $d_{\min }$ show no evidence of systolic anterior motion at the end of the cycle.

In order to complete this test also a complementary simulation with a physiological mitral valve (Fig. 2b) and a pathologic ventricle should be carried out; this case, however, will be considered later (see Sect. 3.6) when assessing the efficacy of a surgical technique called leaflet plication. 

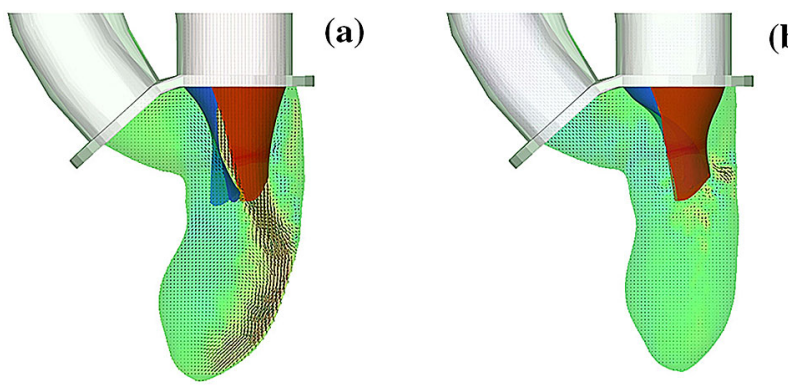

(b)

Fig. 10 The same as Fig. 5, but with a reduced ejection fraction of 40\% (case \#4): a peak of E-wave, b end-systole

Direct measurement of the intraventricular subvalvular pressure drop yields a value $S P D \simeq 4.2 \simeq$ $20.8 \mathrm{mmHg}$ which is below the threshold for SAM to develop, consistently with the results shown in Fig. 9 .

\subsection{Case \#4: Severe hypertrophy, elongated leaflets and reduced ejection fraction}

We have seen that SAM is triggered by the low pressure at the aortic channel induced by the anomalous flow velocity. In the previous sections we have shown that this requires the presence of septum thickening and mitral leaflets elongation and that one of these factors alone is not enough to produce the problem (see also Sect. 3.6). The low pressure in the aortic subvalvular region, however, is generated only if the above geometric factors are combined with a systolic flow rate, or the ejection fraction, strong enough for the flow to abnormally accelerate. It is reasonable to speculate, therefore, that an impaired ventricle is less likely to produce SAM and for the present case we have used the same parameters as Sect. 3.2 except for an ejection fraction of $E F=40 \%$ rather than the physiological value $E F=60 \%$.

A comparison of Figs. 8 and 10 reveals that indeed the flow with a reduced ejection fraction does not generate appreciable SAM since the weak systole produces flow velocities that are not strong enough to decrease the pressure in the aortic subvalvular region below the threshold. Similar information comes from Fig. $7 \mathrm{~b}$ with $\bar{X}$ which is only slightly smaller than the healthy value of Case \#0 and $d_{\min }$ which remains large enough for $S P D(\simeq 27 \mathrm{mmHg}$ ) to stay always (barely) below the pathologic threshold after the valve closure.

3.5 Inclusion of chordae tendineae: medium hypertrophy of the left ventricle and elongated chorded leaflets

In a real left ventricle the chordae tendineae, connecting the leaflet tips with the papillary muscles, are organized in a complex web of filaments that make extremely difficult their precise replica (leaving aside the strong variability among different individuals). Therefore, as already done in [11], we reduce all the chordae to four main bundles: each one is a 'ribbon' with the same material properties as the leaflet tissue, departs from one distal corner of a leaflet and connects directly to the ventricle papillary muscle. As shown in [11] the resulting chorded valve can reproduce the main functionalities of the native mitral valve and, while maintaining the computational cost at an acceptable level, prevents the valve prolapse without any external kinematic constraint. The role of the chordae tendineae in the pathophysiology of HCM is evaluated here by performing a simulation with the same set-up as in Sect. 3.1 except for the mitral valve and left ventricle geometry which are respectively provided with chordae tendineae and papillary muscles. This medium hypertrophic ventricle is characterized by an initial volume $V_{\min }=2.9 D^{3}$ which is about two-thirds of the physiologic value. The mitral valve presents an elongation of the leaflets $\ell=1.20 D$, as sketched in Fig. 2c. In Fig. 11 two snapshots, at the peak of the E-wave $(t=0.15)$ and at the peak systole $(t=0.8)$, with velocity vectors and vertical velocity contours in the symmetry plane are reported showing a dynamics similar to the cases 3.1 and 3.2. Also in this case, as described in Sect. 3.1, after the valve closure $(t \approx 0.75)$ the coapted leaflets show the anterior drift towards the ventricle septum $(t \approx 0.85)$ despite the presence of the chordae. As anticipated before the different pulling of the chordae on the mitral leaflets opens them slightly, however, the SAM is still present and the overall intraventricular flow is only marginally affected by the presence of these additional structures. In order to quantify the obstruction level we compute the subvalvular pressure drop obtaining the value of $S P D \approx 6.2$, corresponding to $\approx 32 \mathrm{mmHg}$, which is close to the one obtained in Sect. $3.1(\simeq 30.5 \mathrm{mmHg})$ and both above the threshold level. As a consequence, also in this case obstruction of the outflow tract is observed. Moreover, 


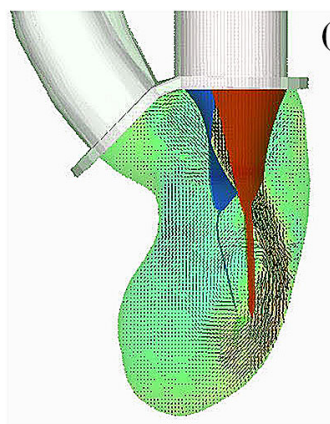

(a)

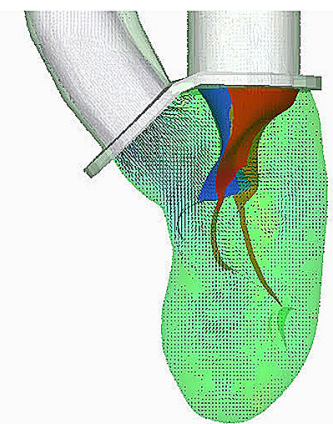

(b)

Fig. 11 The same set-up of Fig. 5, but for a mitral valve with chordae tendineae and a left ventricle with papillary muscles: a peak of E-wave, $\mathbf{b}$ end-systole

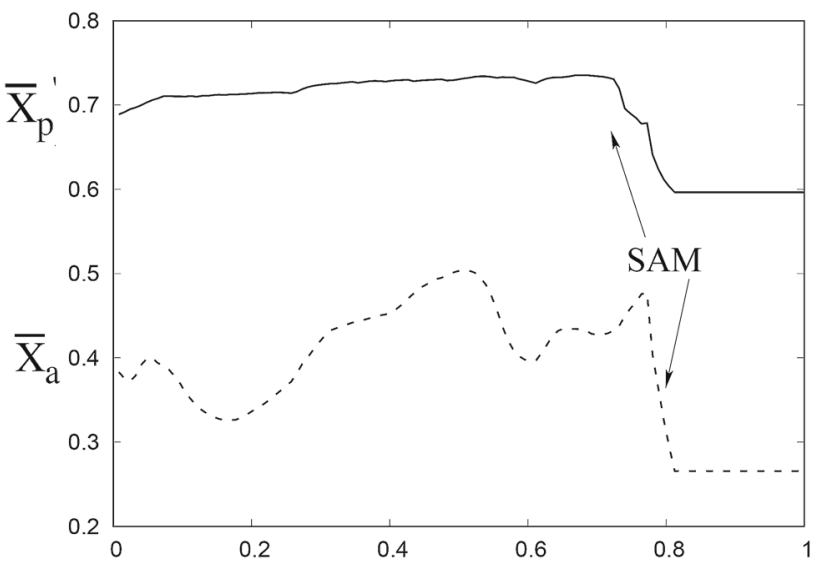

Fig. 12 Time evolution of the anterior $\bar{X}_{\mathrm{a}}$ and posterior $\bar{X}_{\mathrm{p}}$ leaflet centroid for the same set-up of (Case \#1) but with a mitral valve provided of chordae tendineae

the kinematics of both, anterior and posterior leaflets in Fig. 12, clearly evidence this drift through the aortic channel and at the same time point out no relevant differences with respect to the dynamics of the non-chorded valve shown in Fig. 7. We can therefore confirm here that, as extensively discussed in [11], including the chordae makes the computational model more realistic but also more computationally demanding (more than a factor two). This suggests that, depending on the specific focus of the study, it is possible to decide whether or not including them and for this study we have determined that they are not an essential component of the model.

\subsection{Testing surgical techniques for HCM: leaflets plication and septal myectomy}

As mentioned in the introduction, HCM is a disease without a known specific cure, even though drug therapy (essentially beta-blocking agents aimed at reducing the systemic blood pressure that, in turn, decreases the $S P D$ ) works reasonably well for many people, at least in the short- to medium-term [6]. There are, however, patients who do not respond to the drug therapy or develop severe HCM symptoms after a few years on medications and alternative surgical procedures must be considered for them. Mitral leaflets plication and septal myectomy are the most effective procedures; the former consists of shortening the elongated leaflets to their physiological length, while the latter implies the surgical removal of the abnormal growth of the thickened septum protruding inside the ventricle, as shown in Fig. 14a. Both are aimed at eliminating the systolic anterior motion and the obstructive pathology.

These surgical procedures are simulated here by the same simulation model as in the previous sections. The leaflet plication is mimicked using the same configuration as in Sect. 3.2 but with the physiological leaflets of Fig. 2b. Figure 13 reports the snapshots of the velocity field at the same instants as Fig. 8 and the absence of SAM of the mitral leaflet at the end of the cycle (Fig. 13b) is confirmed. Figure 7b yields similar information showing no decrease of the mitral valve $x$-centroid and the distance $d_{\min }$ within a large enough 

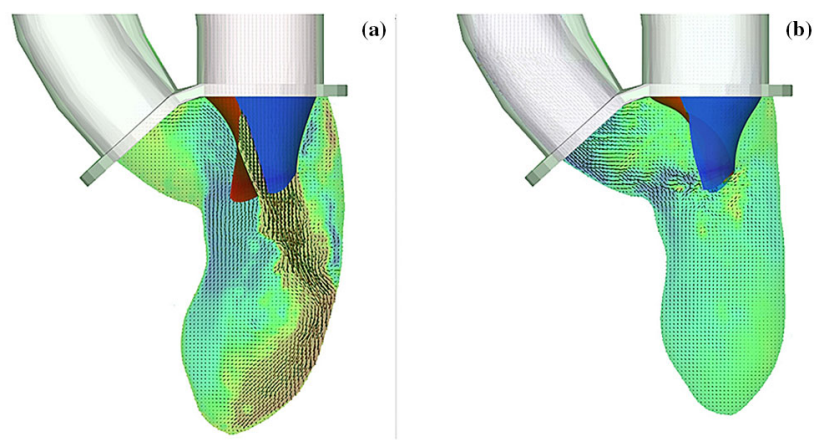

Fig. 13 The same as Fig. 8, but for a mitral valve with leaflets plication: (case \#5): a peak of E-wave, b end-systole
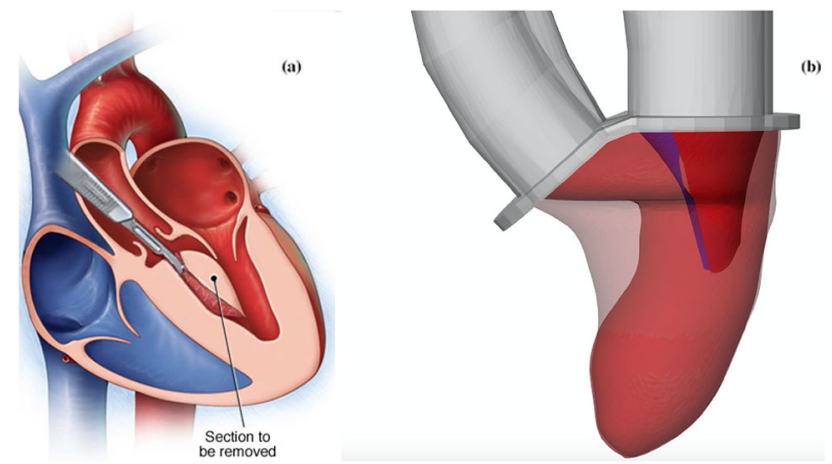

Fig. 14 a Sketch of septal myectomy intervention. b Numerical set-up for the simulation of septal myectomy: the red ventricle geometry is that with severe hypertrophy of Case \#2, the transparent pink geometry represents a ventricle after myectomy

value. Consistently, the subvalvular pressure drop in the present case is $S P D=3.8 \simeq 19 \mathrm{mmHg}$ which is clearly below the pathologic threshold value of (6) to produce SAM.

As mentioned before, this case can be considered also as the complementary test of that described in Sect. 3.3 (Case \#3) since here the ventricle is hypertrophic but the mitral valve is physiologic: Cases \#3 and \#5 together show that only the contemporary presence of hypertrophic growth and elongated leaflets can produce SAM.

The alternative surgical intervention, the septal myectomy, is also numerically simulated and, since it consists of removing the overgrown septum (Fig. 14a), a new left ventricle geometry has been generated and combined with elongated leaflets (Fig. 14b).

The results are summarized in Fig. 15; as for the previous case, the system dynamics is similar to that of Fig. 8 during diastole, whereas no migration of the elongated mitral leaflets is observed during systole. Again the mitral valve centroid $\bar{X}$ does not show an excessively small value and similar indication comes from $d_{\min }$. The subvalvular pressure drop, with a value around $4 \simeq 20 \mathrm{mmHg}$, gives a consistent indication being, also in this case, sufficiently far from the threshold of $S P D=6 \simeq 30 \mathrm{mmHg}$.

In both cases the numerical results confirm the effectiveness of the surgical procedures and this is an additional confirmation of the reliability of the present model which could be used to improve the current intervention techniques and to predict the outcome of innovative procedures or to fine tune the risk stratification of the patients.

\section{Summary and closing remarks}

In this paper we have applied a previously developed computational model to the pathophysiology of hypertrophic cardiomyopathy of the left ventricle. This tool has proven to give not only plausible results but also quantitative reliable information for the flow and the mitral valve dynamics and especially for the SAM. A full parametric study has been performed to assess the role of the factors implicated for systolic anterior motion.

The SAM has been indeed observed in cases with both elongated leaflets and hypertrophic ventricles, at different severity levels of the pathology. The phenomena have shown little sensitivity to the presence of 

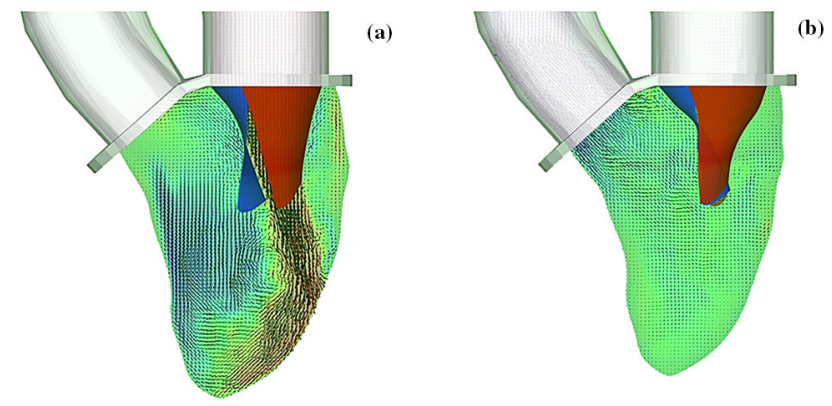

Fig. 15 The same as Fig. 5, but a ventricle geometry mimicking the septal myectomy surgery (case \#6): a peak of E-wave, b end-systole

chordae tendineae since, adding them to the set-up with medium hypertrophy of the left ventricle, has shown the same occurrence of SAM as a case without chordae and no relevant differences in the flow field. In contrast, other factors like the hypertrophy of the intraventricular septum or the mitral leaflet elongation have shown a strong effect on SAM, especially when they act synergistically. For example, neither a physiological ven- tricle with elongated leaflets nor a hypertrophic ventricle with physiologic leaflets could produce the pathological behaviour while their simultaneous presence did it, thus confirming that a single factor alone does not induce SAM. In contrast, a physiological ventricle with elongated leaflets or an hypertrophic ventricle with physiologic leaflets has not shown the pathological behaviour thus confirming that a single factor alone does not induce SAM. In an additional test it has been found that in order for the pathology to be effective, not only the hypertrophy has to be severe and the mitral valve leaflets elongated but also the ventricle ejection fraction must be within physiologic values. In fact a case with $E F=40 \%$ has produced a too weak flow to generate the low subvalvular aortic pressure necessary for SAM. Finally, the two standard surgical procedures of leaflet plication and septal myectomy have been numerically tested and their effectiveness in eliminating the SAM has been assessed.

All the numerical results have indicated that the occurrence of pathological behaviours in HCM are related to the combined effects of thickened and abnormal growth of the septum, elongated leaflets of the mitral valve and physiological values of the ejection fraction; if one of these factors is missing the systolic anterior motion does not occur. A quantitative confirmation of the correct predictions has been obtained from the evaluation of the intraventricular subvalvular pressure drop (or subvalvular pressure gradient for the medical community), which gives an immediate quantification of the systolic anterior motion: when this quantity attains the value of $30 \mathrm{mmHg}$ SAM of the mitral leaflets is observed while when this threshold is exceeded the SAM becomes obstructive. Conversely, when no SAM is observed the pressure drop is always below the threshold. This study is being continued along several lines in order to make the computational model more realistic and to add additional features of the heart dynamics.

\section{References}

1. Boyang, S., et al.: Cardiac MRI based numerical modeling of left ventricular fluid dynamics with mitral valve incorporated. J. Biomech. 26, 225-230 (2016)

2. de Tullio, M.D., Pascazio, G.: A moving least-squares immersed boundary method for simulating the fluid-structure interaction of elastic bodies with arbitrary thickness. J. Comput. Phys. 235, 201-225 (2016)

3. Donato, M.D., et al.: Left ventricular geometry in normal and post-anterior myocardial infarction patients: sphericity index and 'new' conicity index comparisons. Eur. J. Cardiothorac. Surg. 29, 225-230 (2006)

4. Fadlun, E.A., Verzicco, R., Orlandi, P., Mohd-Yosuf, J.: Combined immersed-boundary finite-difference methods for threedimensional complex flow simulations. J. Comput. Phys. 161, 35-60 (2000)

5. Feins, E.N., Yamauchi, H., Marx, G.R., Freudenthal, F.P., Liu, H., del Nido, P.J., Vasilyev, N.V.: Repair of posterior mitral valve prolapse with a novel leaflet plication clip in an animal model. J. Thorac. Cardiovasc. Surg. 147(2), 783-791 (2014)

6. Gregor, P., Curila, K.: Medical treatment of hypertrophic cardiomyopathy-What do we know about it today? Cor et Vasa 57, e219-e224 (2015)

7. Koester, M.: A review of sudden cardiac death in young athletes and strategies for preparticipation cardiovascular screening. J. Athlet. Train. 36(2), 197-204 (2001) 
8. Maron, B.J., Gardin, J.M., Flack, J..M..e.a: Assessment of the prevalence of hypertrophic cardiomyopathy in a general population of young adults: echocardiographic analysis of 4111 subjects in the cardia study. Circulation 92, 785-944 (1995)

9. Maron, B.J., Ommen, S.R., Semsarian, C., Spirito, P., Olivotto, I., Maron, M.S.: Hypertrophic cardiomyopathy. Present and future, with translation into contemporary cardiovascular medicine. J. Am. Coll. Cardiol. 64, 83-99 (2014)

10. Meschini, V., de Tullio, M.D., Querzoli, G., Verzicco, R.: Flow structure in healthy and pathological left ventricles with natural and prosthetic mitral valves. J. Fluid Mech. 834, 271-307 (2018)

11. Meschini, V., de Tullio, M.D., Verzicco, R.: Effects of chordae tendineae on the flow in the left heart ventricle. Eur. Phys. J. E 41(2), 27 (2018)

12. Mittal, R., Seo, J.H., Vedula, V., Choi, Y.J., Liu, H., Huang, H.H., Jain, S., Younes, L., Abrahamd, T., George, R.T.: Computational modeling of cardiac hemodynamics: current status and future outlook. J. Comput. Phys. 305, 1065-1082 (2016)

13. Naidu, S.S.: Hypertrophic Cardiomyopathy. Springer, New York (2015)

14. Seo, J.H., et al.: Effect of the mitral valve on diastolic flow patterns. Phys. Fluids 26, 121901 (2016)

15. Sherrid, M., Balaram, S., Kim, B., Axel, L., Swistel, D.: The mitral valve in obstructive hypertrophic cardiomyopathy. J. Am. Coll. Cardiol. 67(15), 1846-1858 (2016)

16. Siginer, D.A., De Kee, D., Chhabra, R.P.: Advances in the Flow and Rheology of Non-Newtonian Fluids. Elsevier, Amsterdam (1999)

17. Spandan, V., Meschini, V., de Tullio, M.D., Querzoli, G., Lohse, D., Verzicco, R.: A parallel interaction potential approach for large scale simulations of deformable interfaces and membranes. J. Comput. Phys. 348, 567-590 (2017)

18. Stouffer, G., Runge, M., Patterson, C., Rossi, J.: Netter's Cardiology. Theoretical Background and Biological/Biomedical Problems. Elsevier, New York (2018)

19. Suinesiaputra, A., et al.: Statistical shape modeling of the left ventricle: myocardial infarct classification challenge. J. Biomed. Health Inform. 22, 503-515 (2016)

20. Tanaka, M., Wada, S., Nakamura, M.: Computational Biomechanics, Theoretical Background and Biological/Biomedical Problems, vol. 3. Springer, New York (2012)

21. University, S.: https://web.stanford.edu

22. Van Gelder, A.: Approximate simulation of elastic membranes by triangulated spring meshes. J. Graph. Tools 3, 21-41 (1998)

23. Vanella, M., Balaras, E.: A moving-least-squares reconstruction for embedded-boundary formulations. J. Comput. Phys. 228(18), 6617-6628 (2009)

Publisher's Note Springer Nature remains neutral with regard to jurisdictional claims in published maps and institutional affiliations. 